\title{
Correction to: COVID-19 pandemic: impacts on bees, beekeeping, and potential role of bee products as antiviral agents and immune enhancers
}

\author{
Youssef A. Attia ${ }^{1,2,3}$ (1) Gianpaolo M. Giorgio ${ }^{4}$ - Nicola F. Addeo ${ }^{4}$. Khalid A. Asiry ${ }^{1}$. Giovanni Piccolo ${ }^{4}$. \\ Antonino Nizza ${ }^{5}$ Carmelo Di Meo ${ }^{4}$ - Naimah A. Alanazi ${ }^{6}$. Adel D. Al-qurashi ${ }^{1}$ - Mohamed E. Abd El-Hack ${ }^{7}$ (1) . \\ Asmaa F. Khafaga ${ }^{8} \cdot$ Fulvia Bovera $^{4}$
}

Published online: 2 February 2022

(c) Springer-Verlag GmbH Germany, part of Springer Nature 2022

\section{Correction to: Environmental Science and Pollution Research (2022) 29:9592-9605 https://doi.org/10.1007/s11356-021-17643-8}

Funding The Deanship of Scientific Research (DSR) at King Abdulaziz University, Jeddah, Saudi Arabia has funded this project, under grant no. (FP-219-42 H). The authors, therefore, thanks DSR, King Abdulaziz University for financial and scientific supports.

Publisher's note Springer Nature remains neutral with regard to jurisdictional claims in published maps and institutional affiliations.

The original article can be found online at https://doi.org/10.1007/ s11356-021-17643-8.

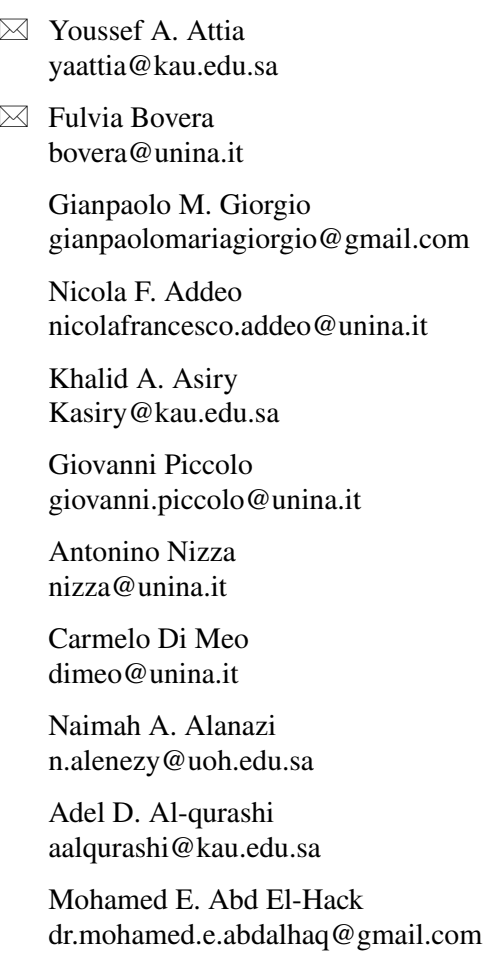

Asmaa F. Khafaga

Asmaa.Khafaga@alexu.edu.eg

1 Agriculture Department, Faculty of Environmental Sciences, King Abdulaziz University, P.O. Box 80208, Jeddah 21589, Saudi Arabia

2 The Strategic Center to Kingdom Vision Realization, King Abdulaziz University, P.O. Box 80200, Jeddah 21589, Saudi Arabia

3 Department of Animal and Poultry Production, Faculty of Agriculture, Damanhour University, Damanhour, Egypt

4 Department of Veterinary Medicine and Animal Production, University of Napoli Federico II, via Delpino, 1, 80137 Napoli, Italy

5 Department of Agronomy, University of Napoli Federico II, Via Università, 100, 80055 Portici, Napoli, Italy

6 Department of Biology, Faculty of Sciences, University of Ha'il, PO Box 2440, Ha'il 81451, Saudi Arabia

7 Poultry Department, Faculty of Agriculture, Zagazig University, Zagazig 44511, Egypt

8 Department of Pathology, Faculty of Veterinary Medicine, Alexandria University, Edfina 22758, Egypt 